\title{
A Novel Video Encryption and Decryption Scheme based on Discrete Wavelet Transform and Fractional Fourier Transform
}

\author{
Vanchhit Goyal \\ M.Tech Scholar \\ Department of Electronics \& Comm. \\ SKIT,Ramnagariya \\ Jaipur, Rajasthan, India
}

\author{
Devesh Mishra \\ Lecturer - Computer Engineering \\ SGV Govt. Polytechnic College \\ Bharatpur,Rajasthan \\ India
}

\author{
Ankit Agarwal \\ Asst.Professor \\ Department of Electronics \& Comm. \\ SKIT,Ramnagariya \\ jaipur,Rajasthan, India
}

\begin{abstract}
We propose three novel algorithms for video encryption and decryption. The algorithms insert one-level of encryption key into the existing methods. Data compression properties of the DWT (Discrete Wavelet Transform) are utilized to make the algorithm faster. The new algorithms retain the robustness of existing image encryption-decryption algorithms.
\end{abstract}

A video is a collection of frames and a frame is similar to an image i.e. it can be segregated into three primary color channels viz. R, G and B. These channels are compressed by these proposed methods by using two times $D W T_{2}$ (2-D Discrete Wavelet Transform). The compressed frame-channels are encrypted using 2-D FRT (The 2-D fractional Fourier transform) and random phase masks in two successive iterations. The encrypted channels are merged by two times application of $I D W T_{2}$ (2-D Inverse Discrete Wavelet Transform), generating a color encrypted frame. Decryption process is the reverse of the encryption process.

Simulations are performed and the results of these simulations verify the proposals made in the new algorithms.

\section{Keywords}

Wavelet Transform, Fractional Fourier Transform, Chaos, Random Phase Mask, Computational Complexity, video encryption.

\section{INTRODUCTION}

Many efficient image encryption-decryption methods have been proposed in recent past [1] - [3]. Video encryption-decryption algorithms are also of growing interest because of their importance in surveillance systems. In the past, a number of such methods have been proposed [4] [5] [6] [7].Efficient image encryption-decryption methods can also be utilized for video (frame) encryption-decryption. As the generalization of the conventional Fourier transform, the fractional Fourier transform has recently shown its potential in the field of optical security $[1]-[3]$.

\section{REVIEW OF FRT}

The $a^{\text {th }}$ order fractional Fourier transform is a linear operation defined by the integral

$$
\begin{gathered}
f_{\alpha}(u) \equiv \int_{-\infty}^{\infty} K_{\alpha}\left(u, u^{\prime}\right) f\left(u^{\prime}\right) d u^{\prime} \\
K_{a}\left(u, u^{\prime}\right) \equiv A_{\alpha} \exp \left[i \pi\left(\cot \alpha u^{2}-2 \csc \alpha u u^{\prime}+\cot \alpha u^{\prime 2}\right)\right] \\
A_{\alpha} \equiv \sqrt{1-i \cot \alpha} \alpha \equiv a \pi / 2
\end{gathered}
$$

When $a \neq 2 j$ and $K_{a}\left(u, u^{\prime}\right) \Xi \delta\left(u-u^{\prime}\right)$ when $a=4 j$ and $K_{a}\left(u, u^{\prime}\right) \Xi \delta\left(u+u^{\prime}\right)$ when $a=4 j \pm 2$, where $j$ is an integer. The $a^{\text {th }}$ order transform is sometimes referred to as the $\alpha^{\text {th }}$ order transform, a practice which will occasionally be found convenient when no confusion can arise [8]

\section{WAVELET TRANSFORM (HAAR WAVELET)}

The wavelet transform is a new mathematical tool developed since the 1980s. It is most efficient for local analysis of non stationary and fast transient wideband signals. The wavelet transform is a mapping of a time division signal to the timescale joint representation that is used in the short-time Fourier transform.

\subsection{Two-Dimensional Wavelet Transform}

The definition of wavelet transform is modified to 2-D case for many 2-D function processing applications. The wavelet transform of a 2-D function $f(x, y)$ is understood as a fourdimensional function.

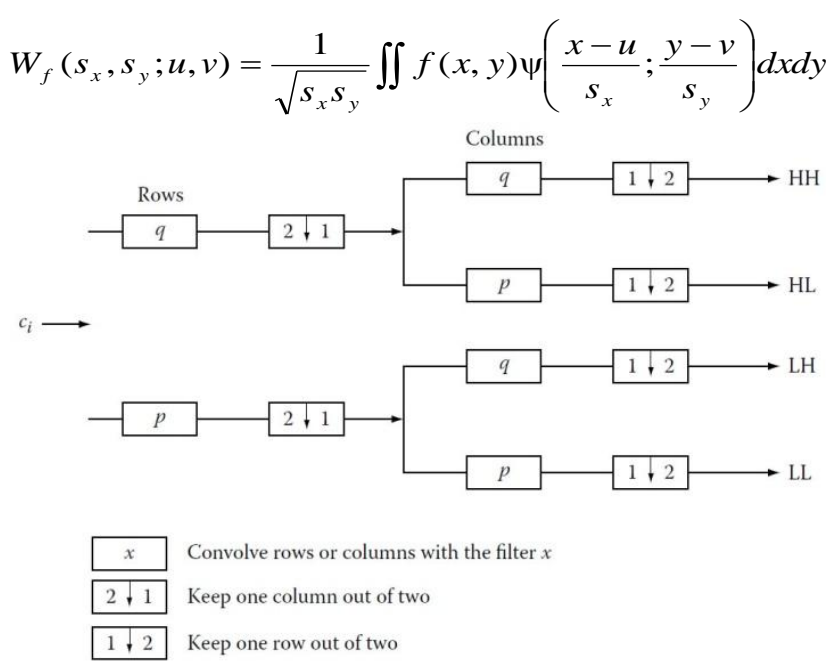

Fig 1 : Schematic two-dimensional wavelet decomposition.

It is reduced to a set of two-dimension functions of $(u, v)$ with different scales, when the scale factors $s_{x}=s_{y}=s$. When $\psi(x$, $y)=\psi(r)$ with $r=\left(x^{2}+y^{2}\right)^{1 / 2}$, the wavelets are isotropic and have no selectivity for spatial orientation. 


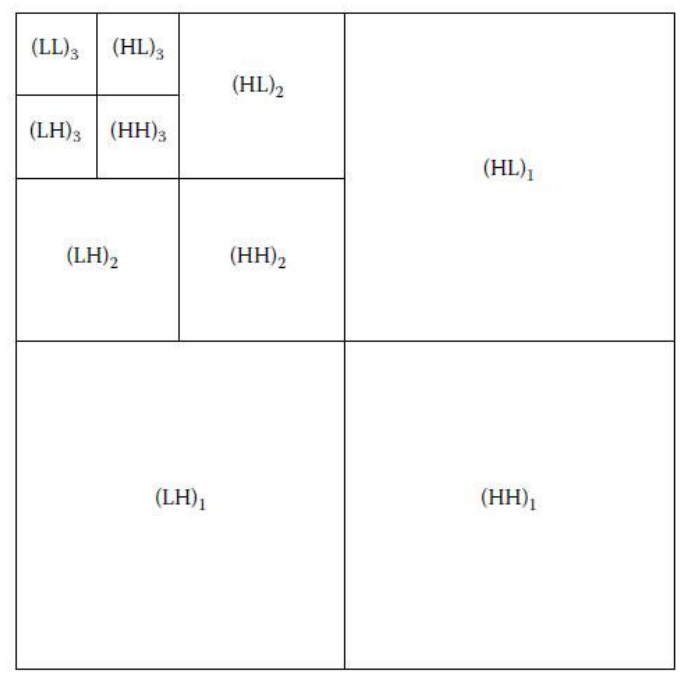

Fig 2: Presentation of the two-dimensional wavelet decomposition and high-pass filters $p(n)$ and $q(n)$.

Otherwise, the wavelet can have particular orientation. The wavelet can also be a combination of the 2-D wavelets with different particular orientations, so that the 2-D wavelet transform has orientation selectivity with quadrature mirror low-pass and high-pass filters $p(n)$ and $q(n)$
Then the pair of the 1-D filters are applied to each column of the two horizontally filtered images. The down-sampling by two is down sampling result in four sub-band images: (LL) for the low-pass filtered both horizontally and vertically image, $(\mathrm{HH})$ for the highpass filtered both horizontally and vertically image, (LH) for lowpass filtered in horizontal direction and high-pass filtered in vertical direction image and (HL) for highpass filtered in vertical direction and high-pass filtered in horizontal direction image, as shown in Figure 2.

\section{PROPOSED FORMULAE}

A video is a sequence $v^{1} v^{2} v^{3} v^{4} \ldots \ldots \mathrm{v}^{m}$ of $m$ frames. Here we are considering that each frame $I=f^{q}(x, y)$ where $1 \leq x, y \leq n$ and $1 \leq q \leq m$ of size $n \times n$ consists of three primary color channels viz. RED, GREEN and BLUE i.e.

$$
f^{q}(x, y)=\sum_{p=1}^{3} f_{p}^{q}(x, y)
$$

$1 \leq p \leq 3$ and $1 \leq q \leq m$, where $m=$ No. of frames in the video

For simplicity, the frame size can be considered to be $n \times n$. We propose two different frame encryption and decryption algorithms. These algorithms use DWT, fractional Fourier transform and chaotic logistic-map and Kaplan-Yorke map. These algorithms follow a similar sequence of operations but they are different in their implementation. The process of encryption and decryption is shown in fig 3 and fig 4 .

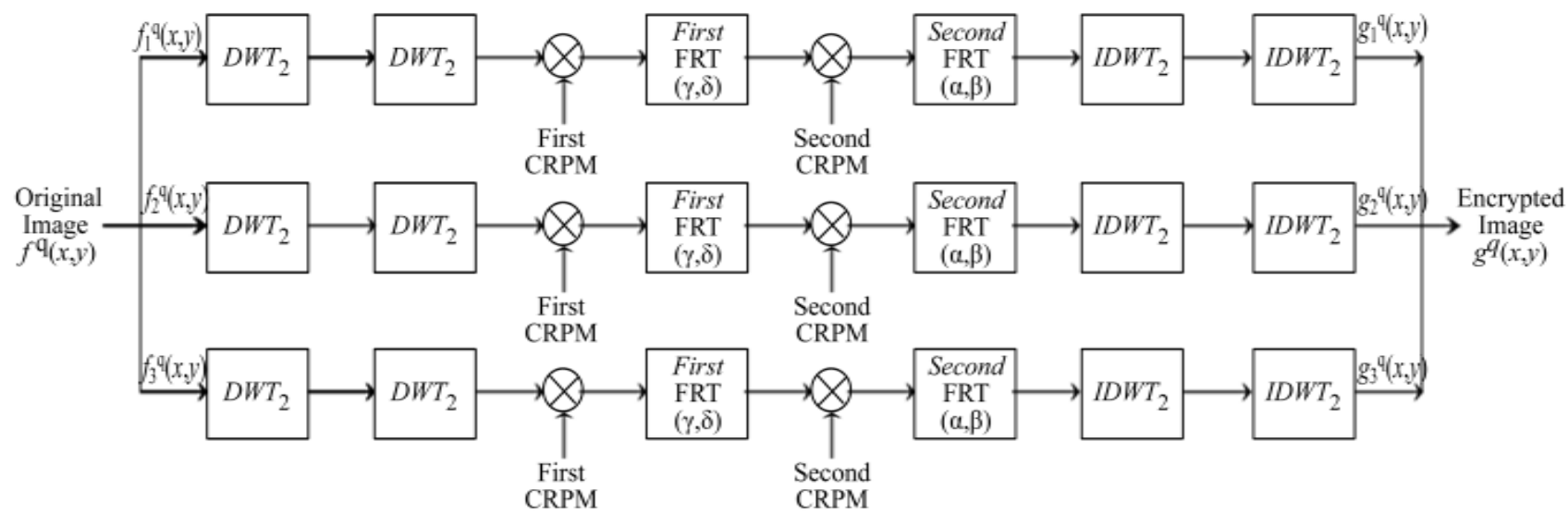

Fig 3: Encryption process using $\mathrm{DWT}_{2}$ in proposed algorithm.

As shown in fig 3, initially, the R, G and B channels of the input frame are segregated. These channels are then concurrently processed by $D W T_{2}$ (twice), $C R P M \& F R T$ (twice) and $I D W T_{2}$ as shown in fig 3 . The processed channels are merged together to produce an encrypted video frame. This process is formulated as below [1].

$$
I_{1}=g^{q}(x, y)=\sum_{p=1}^{3} g_{p}^{q}(x, y)=\sum_{p=1}^{3} I D W T_{2}\left\{I D W T_{2}\left\{F_{\alpha, \beta}\left\{F_{\gamma, \delta}\left\{D W T_{2}\left\{D W T_{2}\left\{f_{p}^{q}(x, y)\right\}\right) * \exp \left(i \frac{\pi}{2} S_{1}(x, y)\right)\right\} * \exp \left(i \frac{\pi}{2} S_{2}(x, y)\right)\right\}\right\}\right\}
$$

where $\alpha, \beta$ are the fractional orders of the second 2-D FRT. $g_{p}{ }^{q}(x, y)$ denotes an encrypted channel viz. R, G or B and $g^{q}(x, y)$ denotes the encrypted frame obtained after merging these channels together.

$$
f^{\prime q}(x, y)=I D W T_{2}\left\{I D W T_{2}\left\{F_{-\gamma,-\delta}\left\{F_{-\alpha,-\beta}\left\{D W T_{2}\left\{D W T_{2}\left\{g_{p}^{q}(x, y)\right\}\right\}\right\} * \operatorname{conj}\left(\exp \left(i \frac{\pi}{2} S_{2}(x, y)\right)\right)\right\} * \operatorname{conj}\left(\exp \left(i \frac{\pi}{2} S_{1}(x, y)\right)\right)\right\}\right\}
$$




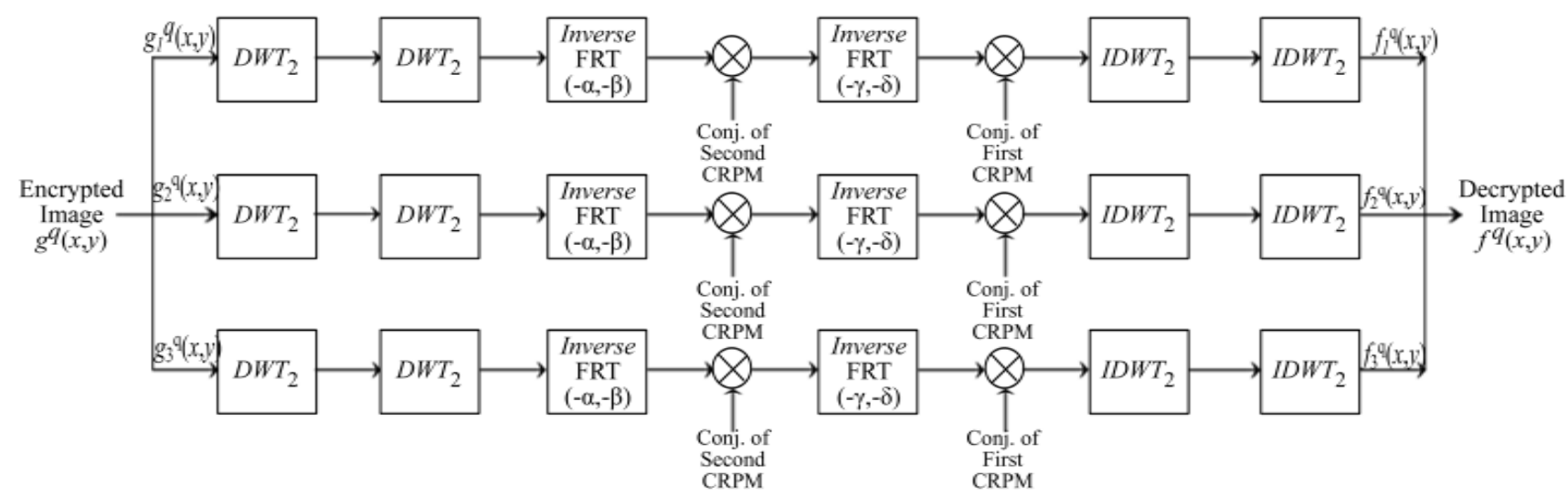

Fig 4: Decryption process using IDWT $_{2}$ in proposed algorithm.

\section{PROPOSED ALGORITHMS}

Algorithm 1: Video encryption and decryption using $\mathrm{DWT}_{2}$ and FRT.

This algorithm uses $D W T_{2}$ and $F_{\alpha, \beta}$ in encryption process and $I D W T_{2}$ and $F_{-\alpha, \beta}$ in decryption process as per the general encryption and decryption schemes Both of the random phase functions are generated as a 2-D sequence of random numbers and they are not chaos based in this algorithm.

\section{Computation complexity:}

Let the input frame $I$ be of size $n \times n$. Analysis of the algorithm is divided into two phases viz. Encryption and Decryption.

Encryption: Frame encryption process involves following steps:

1. Application of $D W T_{2}$ twice on the primary color $\mathrm{R}, \mathrm{G}$, and B channels $f_{p}^{q}(x, y)$ of original frame

2. Encoding by first CRPM.

3. First application of 2-D FRT.

4. Encoding by second CRPM.

5. Second application of 2-D FRT

6. In this last step $I D W T_{2}$ is applied twice on each of the channels obtained after step 5 .

In step 1, each of the primary color channels of original frame is operated twice using $D W T_{2}$, The asymptotic upper bound [9] of this process is $O(n)+O\left(\frac{n}{2}\right)=O(n)$

Thus step 1 takes $O(n)$ time and produces an output frame of size $\frac{n}{4} \times \frac{n}{4}$. Therefore, steps 2 to 6 are to be applied on a smaller sized frame than the original frame.

Computation of 2-DFRT of an frame of size $n \times n$ is a $O\left(2 n^{3}+n \log _{2} n\right)$ process. Also, the generation and multiplication of first and second random phase function is an $O\left(2 n^{2}\right)$ process. But now, as the input frame size for steps 2 to 6 has reduced to $\frac{n}{4} \times \frac{n}{4}$,

(i) Step 2 takes $O\left(2\left(\frac{n}{4}\right)^{2}\right)=O\left(\frac{n^{2}}{8}\right)$ computation time. (ii) Step 3 takes $O\left(2\left(\frac{n}{4}\right)^{3}+\frac{n}{4} \log _{2}\left(\frac{n}{4}\right)\right)=$

$$
O\left(\frac{n^{3}}{32}+\frac{n}{4} \log _{2}\left(\frac{n}{4}\right)\right)
$$

Similar to step 2 and 3, step 4 and 5 also take $O\left(\frac{n^{2}}{8}\right)$ and $O\left(\frac{n^{3}}{32}+\frac{n}{4} \log _{2}\left(\frac{n}{4}\right)\right)$ computation time, respectively. Step 6 involves computation of inverse wavelet transform, which is also a $\boldsymbol{O}(\boldsymbol{N})$ function. Therefore, the computation complexity of encryption is:

$$
\begin{aligned}
T_{\text {encryption }} & =O(N)+O\left(\frac{n^{2}}{8}\right)+O\left(\frac{n^{3}}{32}+\frac{n}{4} \log _{2}\left(\frac{n}{4}\right)\right)+O\left(\frac{n^{2}}{8}\right) \\
& +O\left(\frac{n^{3}}{32}+\frac{n}{4} \log _{2}\left(\frac{n}{4}\right)\right)+O(n) \\
& =O\left(\frac{n^{3}}{16}+\frac{n^{2}}{4}+\frac{n}{2} \log _{2}\left(\frac{n}{4}\right)+2 n\right) \\
\Rightarrow T_{\text {encryption }} & =O\left(\frac{1}{2}\left(\frac{n}{2}\right)^{3}\right)
\end{aligned}
$$

Decryption: Frame decryption process involves following steps:

1. Application of $D W T_{2}$ twice on the primary color components $g_{p}^{q}(x, y)$ of encrypted frame.

2. Application of 2-D inverse FRT.

3. Decoding by conjugate of second CRPM.

4. Application of 2-D inverse FRT.

5. Decoding by conjugate of first CRPM.

6. In this last step $I D W T_{2}$ is applied twice on each of the channels obtained after step 5.

In step 1, each of the primary color channels of encrypted frame is operated twice using $D W T_{2}$, The asymptotic upper bound [9] of this process is $O(n)+O\left(\frac{n}{2}\right)=O(n)$. Thus 
step 1 takes $O(n)$ time and produces an output encrypted frame of size $\frac{n}{4} \times \frac{n}{4}$. Therefore, steps 2 to 6 are to be applied on a smaller sized frame than the original encrypted frame.

(i) Step 2 takes $O\left(2\left(\frac{n}{4}\right)^{3}+\frac{n}{4} \log _{2}\left(\frac{n}{4}\right)\right)$

$=O\left(\frac{n^{3}}{32}+\frac{n}{4} \log _{2}\left(\frac{n}{4}\right)\right)$ time.

(ii) Step 3 takes $O\left(2\left(\frac{n}{4}\right)^{2}\right)=O\left(\frac{n^{2}}{8}\right)^{\text {time. }}$

Similar to step 2 and 3 , step 4 and 5 also take $O\left(\frac{n^{3}}{32}+\frac{n}{4} \log _{2}\left(\frac{n}{4}\right)\right)$ and $O\left(\frac{n^{2}}{8}\right)$ computation time, respectively. Step 6 involves computation of inverse wavelet transform, which is an $\boldsymbol{O}(n)$ process. Therefore, the computation complexity of decryption is:

$$
\begin{array}{r}
T_{\text {decryption }}=O(n)+O\left(\frac{n^{2}}{8}\right)+O\left(\frac{n^{3}}{32}+\frac{n}{4} \log _{2}\left(\frac{n}{4}\right)\right)+O\left(\frac{n^{2}}{8}\right) \\
+O\left(\frac{n^{3}}{32}+\frac{n}{4} \log _{2}\left(\frac{n}{4}\right)\right)+O(n) \\
=O\left(\frac{n^{3}}{16}+\frac{n^{2}}{4}+\frac{n}{2} \log _{2}\left(\frac{n}{4}\right)+2 n\right) \\
\Rightarrow T_{\text {decryption }}=O\left(\frac{1}{2}\left(\frac{n}{2}\right)^{3}\right)
\end{array}
$$

Thus, the computation complexity of algorithm 1 is evaluated to:

$$
T_{1}=T_{\text {encryption }}+T_{\text {decryption }}=O\left(\frac{n}{2}\right)^{3}
$$

There are $\mathrm{m}$ frames is a video therefore the total computation time for encryption $\&$ decryption process is

$$
\begin{aligned}
T_{1} & =O\left(m \frac{n^{3}}{8}\right) \\
\Rightarrow & T_{1} \propto m, T_{1} \propto n^{3}
\end{aligned}
$$

\section{Algorithm 2: Video encryption and decryption using $\mathrm{DWT}_{2}$ and FRT with logistic map.}

This algorithm uses $D W T_{2}, F_{\alpha, \beta}$ in encryption process and $I D W T_{2}, F_{-\alpha,-\beta}$ in decryption process as per the general encryption and decryption schemes shown in Fig. 1 and Fig. 2.

In this algorithm, both of the random phase functions are generated by using logistic map, which is a one-dimensional function.

\section{Computational complexity}

The analysis of this algorithm is also performed in two phases as in case of the Algorithm 1:

Encryption: Step 1, 3, 5 and 6 of encryption are similar to that of algorithm 1 and so are their computation times. Step 2 is Encoding by first random phase function and it takes
$O\left(2\left(\frac{n}{4}\right)^{2}\right)=O\left(\frac{n^{2}}{8}\right)$ time. Similarly step 4 is Encoding by second random phase function and it takes $O\left(\frac{n^{2}}{8}\right)$ time.

Therefore, the computation complexity of entire encryption phase is computed as follows:

$$
\begin{aligned}
T_{\text {Encryption }}=O(n)+O\left(\frac{n^{2}}{8}\right) & +O\left(\frac{n^{3}}{32}+\frac{n}{4} \log _{2}\left(\frac{n}{4}\right)\right)+O\left(\frac{n^{2}}{8}\right) \\
& +O\left(\frac{n^{3}}{32}+\frac{n}{4} \log _{2}\left(\frac{n}{4}\right)\right)+O(n) \\
= & O\left(\frac{n^{3}}{16}+\frac{n^{2}}{4}+\frac{n}{2} \log _{2}\left(\frac{n}{4}\right)+2 n\right) \\
\Rightarrow T_{\text {Encryption }} & =O\left(\frac{1}{2}\left(\frac{n}{2}\right)^{3}\right)
\end{aligned}
$$

Decryption: Steps 1, 2, 4, 6 and their computation times of algorithm 2 are similar to that of the Algorithm 1. Step 3 and 5 perform decoding by conjugate of second and first CRPM respectively and each of them takes $O\left(\frac{n^{2}}{8}\right)$ time. Therefore,

$$
\begin{aligned}
T_{\text {deryption }}=O(n)+O\left(\frac{n^{2}}{8}\right)+ & O\left(\frac{n^{3}}{32}+\frac{n}{4} \log _{2}\left(\frac{n}{4}\right)\right)+O\left(\frac{n}{8}\right) \\
& +O\left(\frac{n^{3}}{32}+\frac{n}{4} \log _{2}\left(\frac{n}{4}\right)\right)+O(n) \\
= & O\left(\frac{n^{3}}{16}+\frac{n^{2}}{8}+\frac{n}{2} \log _{2}\left(\frac{n}{4}\right)+2 n\right) \\
\Rightarrow & T_{\text {Decryption }}=O\left(\frac{1}{2}\left(\frac{n}{2}\right)^{3}\right)
\end{aligned}
$$

Thus, the computational complexity of algorithm 2 is evaluated to:

$$
T_{2}=T_{\text {encryption }}+T_{\text {decryption }}=O\left(\frac{n}{2}\right)^{3}
$$

There are $\mathrm{m}$ frames is a video therefore the total computation time for encryption \& decryption process is

$$
\begin{gathered}
T_{2}=O\left(m \frac{n^{3}}{8}\right) \\
\Rightarrow T_{2} \propto m, T_{2} \propto n^{3}
\end{gathered}
$$

\section{Algorithm 3 - Video encryption and decryption using DWT and FRT with Kaplan-Yorke map.}

This algorithm is similar to the Algorithm 2; the only difference lies in the chaotic function used to generate the random phase mask which is two-dimensional Chaotic Kaplan-Yorke map

The expression for the computational complexity of algorithm 3 is evaluated to:

$$
\begin{gathered}
T_{3}=T_{\text {encryption }}+T_{\text {decryption }}=O\left(\frac{n^{3}}{8}\right) \\
T_{3}=O\left(m \frac{n^{3}}{8}\right) \\
\Rightarrow T_{3} \propto m, T_{3} \propto n^{3}
\end{gathered}
$$




\section{SIMULATION RESULTS}

In order to investigate the quality of encryption, decryption and efficiency of proposed algorithms, digital simulations were performed in an environment as under:

Processor - Intel ${ }^{\circledR}$ Core ${ }^{\mathrm{TM}} 2$ Duo CPU, T5670@1.80GHz.

RAM - 2.00 GB. Operating System -Windows Xp sp2.

Simulation tool - MATLAB $B$ R2008b.

The input video chosen for analysis is nasa_117_256.avi (AVI Video; Size $=283 \mathrm{~KB}$; pixel by pixel resolution $=256 \mathrm{X} 256$; no. of frames =117).The video, nasa_117_256.avi was encrypted using algorithm 1,2 and 3 for $\alpha=\beta=\gamma=\delta=0.5$ fractional orders of $F R T$. The frames encrypted using algorithm 2 and 3 are shown in Fig 6(a) and 6(b). These frames can be decrypted on any fractional order of $F R T$, but the restored frame may differ from the input frame depending on the order of FRT. The decrypted frames obtained using incorrect fractional orders of inverse $F R T$ are shown in Fig 7(a) and 7(b).Fig. 8(a) and 8(b) show decrypted frames obtained when correct fractional orders of inverse FRT are used.

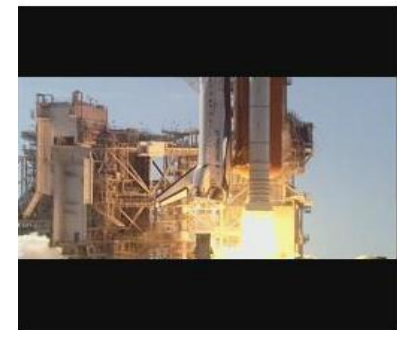

Fig 5 : Input frame to algorithm 1, 2 and 3 (nasa_117_256.avi, 256X256, color).

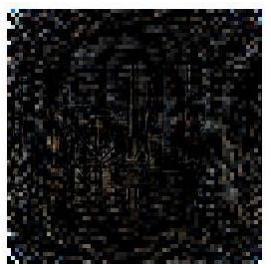

(a)

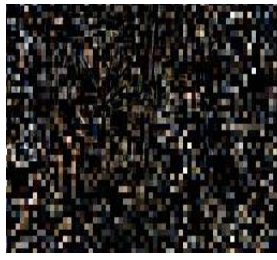

(b)
Fig 6: Encrypted frames of size $256 \times 256$ by (a) Algorithm 2 and (b) Algorithm 3. The fractional orders of FRT are $\alpha=\beta=\gamma$ $=\boldsymbol{\delta}=\mathbf{0 . 5}$.
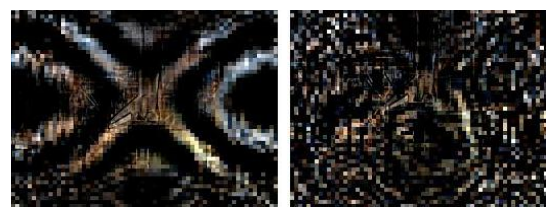

Fig 7 : Decrypted frames of size 256X256 decrypted on an incorrect fractional order of inverse FRT bob(a) Algorithm 2 and (b) Algorithm 3. The fractional orders of $F R T$ are $\alpha=$

$$
\beta=\gamma=\delta=0.4 \text {. }
$$
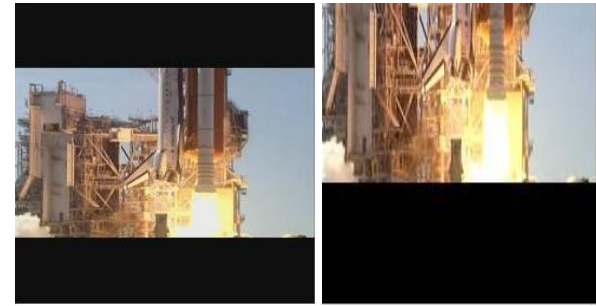

Fig 8 (a) Decrypted frames of size 256X256 decrypted on appropriate fractional order of inverse $F R T$ by (a)

Algorithm 2 and (b) Algorithm 3.The fractional orders of $F R T$ are $\alpha=\beta=\gamma=\delta=0.5$.

Table 1: Computation times of Algo 1, Algo 2 and Algo 3 with no. of frames.

\begin{tabular}{|c|c|c|c|}
\hline \multirow{2}{*}{$\begin{array}{c}\text { No. of } \\
\text { frames } \\
(\mathrm{m})\end{array}$} & \multicolumn{3}{|c|}{ Computation Time (in Seconds) } \\
\cline { 2 - 4 } & Algo 1 & Algo 2 & Algo 3 \\
\hline 1 & 2.71 & 2.80 & 2.79 \\
\hline 3 & 7.39 & 7.62 & 7.62 \\
\hline 7 & 17.08 & 17.45 & 17.34 \\
\hline 14 & 33.85 & 34.72 & 34.69 \\
\hline 29 & 75.73 & 73.25 & 72.88 \\
\hline 58 & 148.66 & 152.51 & 154.28 \\
\hline
\end{tabular}

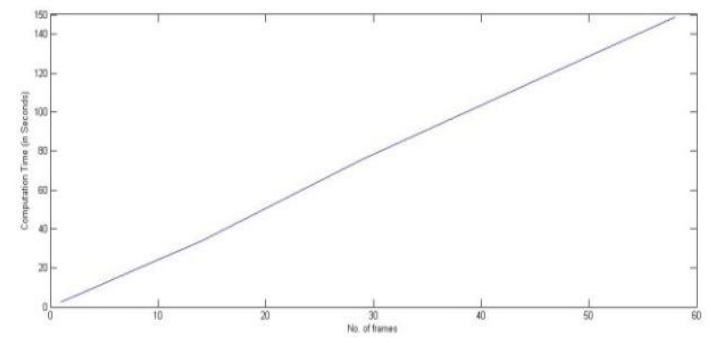

Fig 9 : Graph showing computation time of Algorithm 1.

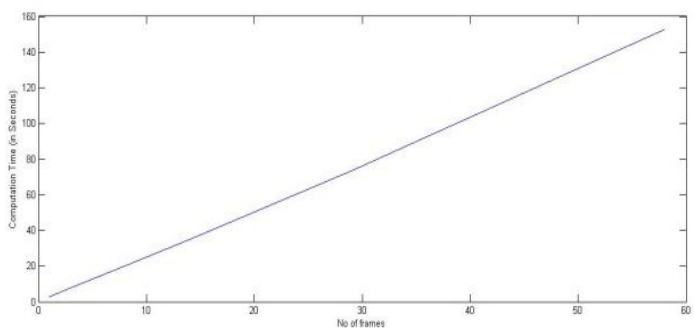

Fig 10 :Graph showing computation time of Algorithm 2.

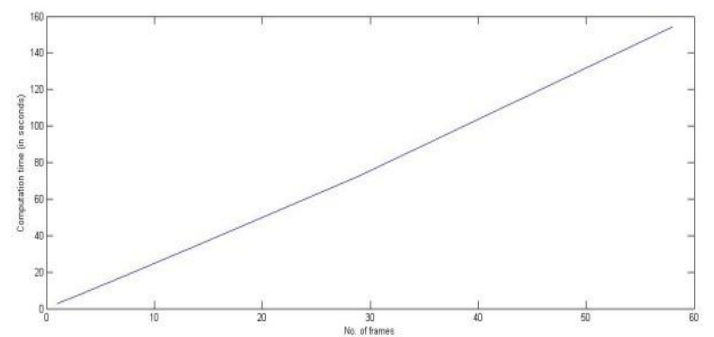

Fig 11: Graph showing computation time of Algorithm 3. 


\section{CONCLUSION}

We have proposed three algorithms for video encryption and decryption and their behavior has been analyzed, based on the computation time required by the algorithms. Each of the algorithm works on a strategy that the data size (or frame size) for encryption and decryption is reduced by a factor of $16(n \mathrm{x}$ $n$ to $\frac{n}{4} \mathrm{X} \frac{n}{4}$ ) than the existing algorithms. For this purpose we have efficiently utilized the data compression characteristics of the discrete wavelet transform.

On the basis of graphs shown in fig 9, 10 and 11 following claims are justified-

$$
T_{1} \propto m, \quad T_{2} \propto m \text { and } T_{3} \propto m
$$

The proposed algorithms retain the robustness of the original image encryption-decryption algorithms. By this we mean that once we encrypt a video frame for a particular fractional order of $F R T$, decryption of this encrypted frame is only possible, when the selected fractional order for decryption is exactly the suitable for decryption. Results are verified for fractional order of 2-D FRT $=0.5$ and fractional order of 2-D IFRT $=0.4,0.5$.

\section{REFERENCES}

[1] Prerana Sharma, Devesh Mishra, AnkurAgarwal, "Efficient Image encryption and decryption using discrete wavelet transform and fractional Fourier transform", Security of Information and Networks, pp 153-157,2012.

[2] Prerana Sharma, Dr. Shiv Kumar, "Efficient Image encryption and decryption using discrete wavelet transform and fractional Fourier transform", M.Tech. Dissertation, Rajasthan Technical University, April 2013.

[3] Prerana Sharma, Dr. Shiv Kumar, "A robust and efficient scheme for image encryption-decryption", IJAIEM, Volume 2, Issue 5, May 2013.

[4] Jolly shah, Dr. Vikas Saxena "Video Encryption : A Survey", International Journal of computer science Issues (IJCSI) Vol. 8,Issue 2, March 2011.

[5] M. Abomhara, Omar Zakaria, Othman o. Khalifa "An Overview of Video Encryption Techniques ", International Journal of Computer Theory and Engineering,Vol. 2,No. 1 February 2010.

[6] Narsimha Raju C, Uma Devi Ganugula, Kannan Srinathan, C.V. Jawahar "Fast and Secure Real Time Video
Encryption", Indian Conference on Computer Vision, Graphics and Image Processing(ICVGIP),pp 257264,December 2008.

[7] Narsimha Raju C, Uma Devi Ganugula, Kannan Srinathan, C.V. Jawahar "A Novel Video Encryption Technique Based on Secret Sharing",ICIP, pp 3136-3139, October 2008.

[8] H. M. Ozaktas, M. AlperKutay, Z. Zalevsky, The fractional Fourier transform: with applications in optics and signal processing, New York, John Wiley \& Sons, 2001 .

[9] T. H. Cormen, C. E. Leiserson, R. L. Rivest, C. Stein, "Growth of Functions", Introduction to algorithms, $3^{\text {rd }}$ Ed., Cambridge, MIT Press, 2009, pp 43 - 64

[10] Y. Zhang, F. Zhao, "The algorithm of fractional Fourier transform and application in digital image encryption", International Conference on Information Engineering and Computer Science, 2009, pp 1 - 4.

[11] R. C. Gonzalez, R. E. Woods, S. L. Eddins, Digital Image processing using MATLAB, Delhi, Pearson Education, 2004.

[12] A. D. Poularikas, "Transforms And Applications Handbook", $3^{\text {rd }}$ ed., New York, CRC Press, 1996.

[13] dwt2, "Wavelet Toolbox",http://www.mathworks.in/help/ toolbox/wavelet/ref/dwt2.html, [January 2012].

[14] Y. Sheng, D. Roberge, and H. Szu, "Optical wavelet transform", Optical Engineering, Vol. 31 Issue 9, 1992, pp $1840-1845$.

[15] H. Szu, Y. Sheng, and J. Chen, "The wavelet transform as a bank of matched filters", Applied Optics, Vol. 31 Issue 17, 1992, pp 3267-3277.

[16] M. O. Freeman, "Wavelet signal representations with important advantages", Optics \& Photonics News, Vol. 4 Issue 8, August 1995, pp 8-14.

[17] J. M. Combes, A. Grosmann, and P. Tchamitchian, "Wavelets", $2^{\text {nd }}$ ed., Berlin,Germany, Springer-Verlag, 1990.

[18] R. C. Alligood, T. D. Sauer, J. A. Yorke, "Chaos; an introduction to dynamical systems", New York, Sringer, 2001. 